\title{
Repetitive transcranial magnetic stimulation versus botulinum toxin injection in chronic migraine prophylaxis: a pilot randomized trial
}

\author{
Hatem S Shehata \\ Eman H Esmail \\ Ahmad Abdelalim \\ Shaimaa El-Jaafary \\ Alaa Elmazny \\ Asmaa Sabbah \\ Nevin M Shalaby
}

Neurology Department, Faculty of Medicine, Cairo University,

Cairo, Egypt
Correspondence: Hatem S Shehata Faculty of Medicine, Cairo University, Cairo, Egypt

Email samirhatem@hotmail.com
This article was published in the following Dove Press journal:

Journal of Pain Research

7 October 2016

Number of times this article has been viewed

Background: Chronic migraine is a prevalent disabling disease, with major health-related burden and poor quality of life. Long-term use of preventive medications carries risk of side effects.

Objectives: The aim of this study was to compare repetitive transcranial magnetic stimulation (rTMS) to botulinum toxin-A (BTX-A) injection as preventive therapies for chronic migraine. Methods: A pilot, randomized study was conducted on a small-scale sample of 29 Egyptian patients with chronic migraine, recruited from Kasr Al-Aini teaching hospital outpatient clinic and diagnosed according to ICHD-III (beta version). Patients were randomly assigned into two groups; 15 patients received BTX-A injection following the Phase III Research Evaluating Migraine Prophylaxis Therapy injection paradigm and 14 patients were subjected to 12 rTMS sessions delivered at high frequency $(10 \mathrm{~Hz})$ over the left motor cortex (MC, M1). All the patients were requested to have their 1-month headache calendar, and they were subjected to a baseline 25-item (beta version) Henry Ford Hospital Headache Disability Inventory (HDI), Headache Impact Test (HIT-6), and visual analogue scale assessment of headache intensity. The primary efficacy measures were headache frequency and severity; secondary measures were 25-item HDI, HIT-6, and number of acute medications. Follow-up visits were scheduled at weeks 4, 6 , 8,10 , and 12 after baseline visit.

Results: A reduction in all outcome measures was achieved in both the groups. However, this improvement was more sustained in the BTX-A group, and both the therapies were well tolerated.

Conclusion: BTX-A injection and rTMS have favorable efficacy and safety profiles in chronic migraineurs. rTMS is of comparable efficacy to BTX-A injection in chronic migraine therapy, but with less sustained effect.

Keywords: chronic migraine, botulinum toxin-A, rTMS, prophylaxis

\section{Introduction}

Chronic migraine is the most common type of chronic headache; it affects $1.4 \%-2.2 \%$ of the general population. ${ }^{1}$ In an Egyptian study, the 1-year prevalence of chronic migraine was $2.9 \%{ }^{2}$ It has a higher impact on health-related quality of life (QoL) due to workday loss and reduced productivity. ${ }^{3}$

Cortical spreading depression (CSD) and trigeminovascular activation are the principal pathophysiological mechanisms of aura and migraine pain, respectively., CSD is potentially the first step as it can activate the trigeminovascular system. ${ }^{6}$ Many studies found evidence of cortical hyperexcitability or impaired intracortical inhibition in migraineurs. ${ }^{7-9}$ The chronicity of migraine was also attributed to changes in cortical 
excitability due to more impairment of central inhibition. ${ }^{10}$ Cortical neuromodulation of the prefrontal and $\mathrm{MC}$ has been shown to be effective in many pain states. ${ }^{11}$

Repetitive transcranial magnetic stimulation (rTMS), with its effect on cortical excitability, could be a potential therapeutic approach for migraine. ${ }^{12}$ The current Food and Drug Administration-approved treatment of chronic migraine is Botulinum toxin-A (BTX-A) injection, and no new disease-modifying drugs have come into clinical practice since the approval of topiramate and BTX. ${ }^{13}$ There is thus clearly a need for more effective pathophysiologically targeted treatment strategies.

The aim of the current study was to compare the effectiveness and safety of BTX-A, being the currently approved standard treatment of chronic headache, to rTMS. To the best of our knowledge, no previous study has addressed this question.

\section{Methods}

This open-label, randomized study was conducted on 29 patients who were diagnosed with chronic migraine according to The International Classification of Headache Disorders - third edition-III (beta version). ${ }^{14}$ They were recruited from Kasr Al-Aini hospital outpatient headache disorders clinic, Cairo University, in the period from June 2013 to March 2015. Patients were randomly assigned to one of two groups; one group received BTX-A $(n=15)$ and the other one received rTMS sessions $(n=14)$. The study was approved by Neurology Department Board in Cairo University, and follows the principles outlined in the Declaration of Helsinki. Written informed consent was obtained from all patients prior to the commencement of the study after a structured interview clarifying the aim and steps of the study.

\section{Exclusionary criteria}

Patients with headache caused by overuse of medication, other chronic primary/secondary headaches, use of headache prophylaxis medication within 4 weeks of baseline, comorbid psychiatric disorders (including depression Beck's Depression Inventory ${ }^{15}$ score $>17$ at day 1 of baseline), symptomatic headache, "demonstrable structural lesion by brain magnetic resonance imaging (MRI)," patients who responded to medical treatments, and those with the possibility of lack of coherence during follow-up period. Prior to administration of study treatment, women in childbearing period should have a negative urine pregnancy test and have been using a reliable means of contraception.

\section{Screening phase}

All the patients were subjected to history taking, clinical assessment, and brain MRI in order to diagnose and to exclude secondary causes. Thereafter, they were requested to complete a baseline, pretreatment headache diary for 1 month in order to assess for headache days/month; attack frequency, duration, severity, characteristics, precipitants, and associated symptoms; and number of weekly acute medications. Initially, 34 patients were recruited, three of them were excluded as they had difficulty in complying with the follow-up visits (they were from northern Egypt), one patient withdrew his informed consent, and one patient failed to complete the screening questionnaire (Figure 1).

\section{Baseline assessment}

After completing headache diary for a period of 1 month, patients were subjected to a baseline 25-item Henry Ford Hospital Headache Disability Inventory (HDI), ${ }^{16}$ Headache

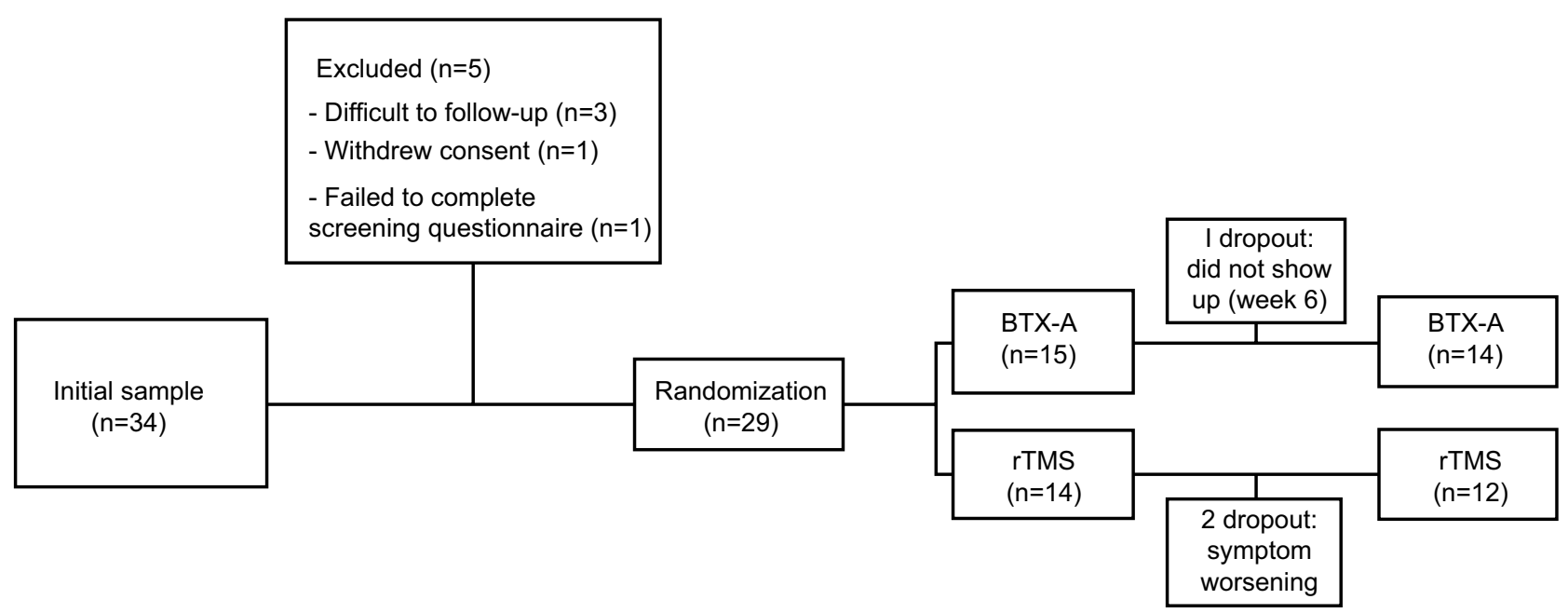

Figure I Study structure and patient flow.

Abbreviations: BTX-A, botulinum toxin-A; rTMS, repetitive transcranial magnetic stimulation. 
Impact Test (HIT-6), ${ }^{17}$ and visual analogue scale (VAS) assessment of headache intensity. ${ }^{18}$

\section{Randomization}

Patients were then randomly assigned to one of two groups using a computer-based randomization; one group received BTX-A and the other group received rTMS sessions. In BTX-A group $(n=15)$, Phase III Research Evaluating Migraine Prophylaxis Therapy injection paradigm was adopted, ${ }^{19}$ with a total of $155-195$ (Botox ${ }^{\circledR}$ ) units were injected in a total of 31 sites across seven specific head and neck muscles \pm 8 sites (following the pain). BTX-A was diluted with $2 \mathrm{~mL}$ of preservative-free normal saline, resulting in a concentration of $5 \mathrm{U} / 0.1 \mathrm{~mL}$. In the rTMS group $(\mathrm{n}=14), 20$ trains (10-s apart) of 100 stimuli each delivered at high frequency $(10 \mathrm{~Hz})$ and $80 \%$ of motor threshold (MT), using figure-of-8-shaped coil over the left MC (M1), were delivered to each patient, 3 days a week, for 1 month. The resting MT for the right abductor pollicis brevis muscle was determined using electromyography. MT intensity was defined as the lowest stimulation intensity that, in 10 trials, induced at least five motor-evoked potentials of at least 50 $\mu \mathrm{V}$ peak-to-peak amplitude. ${ }^{20}$ A Magstim Rapid ${ }^{\circledR}$ magnetic stimulator (Magstim Co. Ltd, Whitland, Dyfed, UK) was used, and the maximal stimulator output "peak magnetic field" was $1.2 \mathrm{~T}$.

Follow-up visits were scheduled at weeks 4, 6, 8, 10, and 12 after baseline visit. In each visit, the headache diary was reviewed by an independent rater in order to assess for headache frequency, severity, and attack-aborting medications. 25-Item HDI and HIT-6 were assessed monthly (at weeks 4,8 , and 12).

\section{Outcome measures}

The primary efficacy measures were headache frequency (days/month) and headache severity assessed by VAS; secondary efficacy measures were 25-item HDI, HIT-6, and number of acute medications.

\section{Safety measures}

Any adverse event that a subject reported during the study was recorded by the investigators, graded for severity (mild, moderate, or severe) and assessed for its relationship to study treatment (none, possible, probable, or definite). A serious adverse event was defined as one that was fatal, lifethreatening, permanently disabling, or required admission to hospital.

\section{Statistical methods}

Data management was carried out with the Statistical Package for Social Sciences (version 17, SPSS Inc., Chicago, IL, USA). Simple descriptive analysis in the form of range, mean \pm standard deviations, and frequencies (number of cases) were calculated for numerical data, and qualitative data were described using percent distribution. Efficacy and safety measures were assessed for all patients based on intention to treat. Comparison of BTX-A and rTMS groups in efficacy measures at endpoints using the LOCF was conducted using unpaired Student's $t$-test, while the differences between means of the variables from same group before and after intervention were assessed using paired $t$-test. The chi-square test was used for comparison between the two groups of categorical data or frequency of events. $P<0.05$ was deemed as significant difference.

\section{Results \\ Clinical characteristics}

A total of 29 Egyptian patients were allocated to either rTMS sessions or BTX-A injection. Their age ranged from 21 to 52 years (mean age: $32.65 \pm 7.82$ years). They were 10 males (34.5\%) and 19 females (65.5\%), and the mean duration of migraine was $5.22 \pm 3.15$ years (ranged from 4 to 11 years). The basic clinical data for both the groups are summarized in Table 1.

\section{Intervention}

In BTX-A arm, the mean injected dose was $176.33 \pm 16.85$ units (Botox $\left.{ }^{\circledR}\right)$, one patient failed to show up after week 6

Table I Basic clinical data of included patients

\begin{tabular}{|c|c|c|c|}
\hline Parameters & $\begin{array}{l}\text { BTX-A group } \\
(n=15)\end{array}$ & $\begin{array}{l}\text { rTMS group } \\
(n=14)\end{array}$ & P-value \\
\hline Age range (mean $\pm S D)$ & $32.81 \pm 7.64$ & $31.98 \pm 8.12$ & 0.85 \\
\hline Sex: F/M & $10 / 5$ & $9 / 5$ & 0.95 \\
\hline $\begin{array}{l}\text { Duration of migraine } \\
\text { (years) }\end{array}$ & $5.12 \pm 2.98$ & $5.6 I \pm 3.20$ & 0.92 \\
\hline Duration of chronicity & $2.38 \pm 1.36$ & $2.55 \pm 1.21$ & 0.41 \\
\hline Headache days/month & $19.31 \pm 3.75$ & $|8.74 \pm 4.2|$ & 0.59 \\
\hline Attack duration (h) & $12.01 \pm 1.35$ & 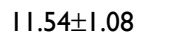 & 0.35 \\
\hline $\begin{array}{l}\text { Headache severity } \\
\text { (VAS) }\end{array}$ & $7.77 \pm 1.54$ & $7.65 \pm 1.65$ & 0.73 \\
\hline $\begin{array}{l}\text { Number of days with } \\
\text { acute medications/mo }\end{array}$ & $11.91 \pm 2.67$ & $12.15 \pm 2.89$ & 0.29 \\
\hline HIT-6 & $63.4 I \pm 4.28$ & $63.53 \pm 4.55$ & 0.96 \\
\hline HDI & $67.12 \pm 6.98$ & $66.45 \pm 7.11$ & 0.88 \\
\hline
\end{tabular}

Abbreviations: F, female; HDI, Headache Disability Inventory; HIT-6, Headache Impact Test; M, male; SD, standard deviation; VAS, visual analogue scale; $h$, hours. 
visit. In the rTMS arm, a total of 24,000 pulses were delivered to each patient over 12 sessions (sessions duration: $6.5 \mathrm{~min}$ ), two patients withdrew their consent during the stimulation period due to worsening headache (Figure 1).

\section{Primary outcome measures}

Ten patients in BTX-A group (66.7\%) achieved a 50\% reduction in their headache frequency and 11 patients $(73.3 \%)$ showed $75 \%$ reduction in headache severity by the end of third week of injection session. In rTMS group, 10 patients (71.4\%) reported $75 \%$ reduction of both headache frequency and severity after $4-5$ sessions.

There was a significant reduction of the headache frequency (headache days per month) and headache severity (VAS) in the first follow-up visit (week 4) as compared to the month before treatment in both BTX-A and rTMS groups; with no significant difference between both arms $(P=0.84)$. This significant improvement was maintained in visit 2 (week 6) and visit 3 (week 8). In the subsequent two visits (week 10 and week 12), this significant difference was maintained in BTX-A arm $(P<0.02$ and 0.03 , respectively); whereas in rTMS arm, the difference in both the parameters (headache frequency and severity) became nonsignificant ( $P=0.07$ and 0.09 , respectively) (Figure 2).

\section{Secondary efficacy measures}

There was a significant improvement of the QoL assessed by HDI and reduction in headache disability assessed by HIT-6 in the first follow-up visit (week 4) as compared to the month before treatment in both BTX-A and rTMS groups, with no significant difference between both the arms $(P=0.62)$. This significant improvement was maintained through week 8 visit. At the last assessment visit (week 12), this significant difference was maintained in BTX-A arm, whereas in rTMS arm, the difference in both the parameters (HDI and HIT-6) became nonsignificant ( $P=0.06$ and 0.21 , respectively) (Table 2 ).

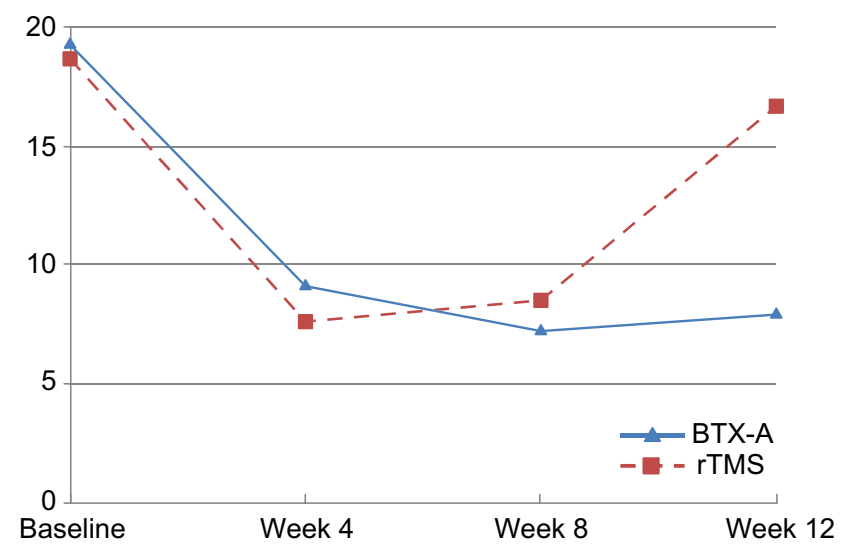

Figure 2 Headache days per month in follow-up period of both the groups. Abbreviations: BTX-A, botulinum toxin-A; rTMS, repetitive transcranial magnetic stimulation.

\section{Safety measures}

No systemic reactions or serious adverse events were recorded. Injections-related adverse events included pain at the site of injection $(\mathrm{n}=5)$, hematoma $(\mathrm{n}=2)$, and blepharoptosis $(n=1)$; these adverse effects were transitory and did not interfere with the patient activity, and did not need further management. In rTMS group ( $\mathrm{n}=14)$, two patients (14.29\%) experienced headache worsening which compelled them to withdraw their consent and one patient (7.14\%) had transient tinnitus on the day of session which lasted for few hours and waned the continuation of sessions.

\section{Discussion}

There is robust evidence that supports the role of rTMS in migraine prevention. Studies have demonstrated that rTMS sessions over the M1 region help to restore defective intracortical inhibition (ICI) and to normalize excitability in the brains of migraineurs. ${ }^{21}$ The efficacy of high-frequency rTMS applied to the dorsolateral prefrontal cortex (DLPFC) was initially demonstrated in a small sham-controlled pilot study. ${ }^{22}$ The postulated mechanism of the excitatory effects

Table 2 Secondary efficacy variables in the studied groups

\begin{tabular}{|c|c|c|c|c|c|}
\hline \multirow[t]{2}{*}{ Efficacy variables } & & \multicolumn{2}{|l|}{ BTX-A } & \multicolumn{2}{|l|}{ rTMS arm } \\
\hline & & Mean (SD) & $\begin{array}{l}\text { P-value (comparison } \\
\text { with baseline) }\end{array}$ & Mean (SD) & $\begin{array}{l}\text { P-value (comparison } \\
\text { with baseline) }\end{array}$ \\
\hline 25-Item Henry Ford & Baseline & $75.87(4.3 \mathrm{I})$ & & $74.93(3.25)$ & \\
\hline Hospital Headache & Week 4 & $40.2 I(5.4 I)$ & 0.002 & $39.57(4.72)$ & 0.01 \\
\hline Disability Inventory & Week 8 & $36.76(8.47)$ & 0.001 & $42.89(4.54)$ & 0.04 \\
\hline (HDI) & Week 12 & $34.80(8.13)$ & 0.001 & $69.31(5.6 I)$ & 0.06 \\
\hline \multirow[t]{4}{*}{ HIT-6 } & Baseline & $63.53(4.55)$ & & $63.41(4.28)$ & \\
\hline & Week 4 & $44.4 I(5.62)$ & 0.03 & $44.29(5.37)$ & 0.03 \\
\hline & Week 8 & $42.86(4.55)$ & 0.01 & $47.47(7.35)$ & 0.04 \\
\hline & Week 12 & $44.27(5.18)$ & 0.02 & $57.33(4.77)$ & 0.21 \\
\hline
\end{tabular}

Abbreviations: BTX-A, botulinum toxin-A; HDI, Headache Disability Inventory; HIT-6, Headache Impact Test; rTMS, repetitive transcranial magnetic stimulation; SD, standard deviation. 
of high frequency sessions in pain modulation was through the connectivity of DLPFC with pain processing centers in the brainstem and thalamus. In a randomized, double-blind, placebo-controlled trial, high-frequency $(10 \mathrm{~Hz}) \mathrm{rTMS}$ delivered to the hot spot of the right abductor digiti minimi provided $>50 \%$ significant reduction in headache frequency and severity with a significant improvement in functional disability when compared to sham treatment. ${ }^{23}$ In another study, Teepker et $\mathrm{al}^{24}$ showed that low-frequency rTMS caused nonsignificant reduction of headache frequency when compared to the sham-treated group. On the contrary, Teo et $\mathrm{al}^{25}$ found that $10 \mathrm{~Hz}$ rTMS over M1 is poorly tolerated by chronic migraine patients, with high dropout rate $(50 \%)$; however, the number of studied subjects was too small for any conclusion. Scalp discomfort and headaches have commonly been reported in rTMS studies, occurring in up to $40 \%$ of cases. $^{26}$

In our series, each session was composed of 2,000 pulses delivered at $10 \mathrm{~Hz}$ over MC. This was adopted according to Brighina et $\mathrm{al}^{21}$ assumption, who reported that the motor ICI is significantly lower in migraineurs with subsequent paradoxical increase of intracortical facilitation (ICF). They also found that $1-\mathrm{Hz}$ stimulation reduced motor-evoked potential amplitude and ICF in healthy controls, whereas it caused a significant ICF increase in migraineurs and showed that high frequency $(10 \mathrm{~Hz})$ stimulation of $\mathrm{MC}$ could potentiate ICI and normalize the cortical excitability through increase in short ICI. On the other hand, the concept of generalized cortical inter-ictal hyper-excitability (mainly in visual cortex) ${ }^{27}$ that was proposed in migraine is controversial; as some authorities documented that the MT, a parameter used to estimate MC excitability, was even higher in migraineurs suggesting cortical hypoexcitability. ${ }^{28}$ Though, there is no single model of migraine that explains all of the known features of the disorder; yet, altered functional connectivity between periaqueductal gray and cortical (limbic) centers plays an important role in migraine expression. ${ }^{29}$ Transcranial magnetic stimulation (TMS) has been used in migraine patients to test occipital cortex excitability by measuring phosphene threshold (PT), defined as the minimum intensity of a TMS pulse needed to evoke phosphenes, which is inversely related to the overall level of visual cortex excitability. ${ }^{30}$ Important discrepancies among different studies do exist, with some reports found increased, and others found decreased in the inter-ictal PT. These conflicting results make it very difficult to reach a definite conclusion by simple summation of previous results. ${ }^{31}$ In the current series, rTMS sessions were applied on motor area (MC). Positive results of MC stimulation and the absence of significant benefits of active high-frequency rTMS of the DLPFC in other studies point to MC as a more promising target than the DLPFC, for larger trials of noninvasive brain stimulation in patients with chronic migraine. ${ }^{32}$ Highfrequency stimulation of both MC and DLPFC can result in an analgesic benefit; however, their relative mechanisms are different ${ }^{33}$; while stimulation at the MC activates a strong focal activation in thalamus, insula, cingulate-orbitofrontal junction, and periaqueductal gray (PAG) area, suggesting a top-down activation of the descending pain control system mediated via a motor-thalamus functional linkage ${ }^{34}$; on the other hand, rTMS at the DLPF exerts a top-down inhibitory effect along the ascending midbrain-thalamic-cingulate pathway through the descending fibers from the prefrontal cortex..$^{35,36}$

Our results revealed significant improvement of both primary and secondary outcomes in both the study arms; however, this improvement was recorded earlier in rTMS group (after 4-5 sessions), but it wanes faster ( 2 weeks after discontinuation of sessions); on the contrary, in the BTX-A arm, significant improvement was recorded by the end of third week and was maintained till the end of the study period (12 weeks). Although a long-term maintenance of analgesia induced by high-frequency rTMS was established by Hodaj et $a 1,{ }^{37}$ in patients with chronic facial pain, yet they did not include chronic migraine and they used different protocols, with induction phase and maintenance phase for $>5$ months. However, this study provided an important evidence of "timelocked" effects of rTMS, as authors noticed significantly lower analgesic effect when session duration was shortened; in the present series, the long-term effects of rTMS was not addressed. The most commonly reported adverse events of rTMS in our patients were headache worsening (14.29\%) and transient tinnitus (7.14\%); in one study, headache was reported in $42 \%$ of participants who received active rTMS and in $33 \%$ of whom had sham sessions, ${ }^{38}$ and this headache was explained by pressing the coil against subjects' heads for extended periods or by the induced muscle contractions. Most are mild and respond to over-the-counter treatments. Other reported adverse events included pain at the stimulation site, neck pain, muscle aches, dizziness, nausea, tiredness, and tinnitus ${ }^{39}$; however, these adverse events are no more common after real TMS than after sham TMS. ${ }^{38}$ Lastly, as for MRI, patients should wear earplugs in order to minimize noise exposure from coil discharge and thus reduce the risk of transient threshold shifts or hearing loss.

A number of limitations should be considered while addressing the results of this study. One concern is the relatively 
small sample size; however, this is a pilot study that provides a preliminary sketch for the feasibility and effectiveness of rTMS in chronic migraine; yet a well-structured protocol for larger randomized controlled trials is highly needed. Other limiting factors are the absence of "placebo" arm and the lack of longterm study of rTMS in chronic migraine. To reduce the influence of absence of placebo, a compromised clinical context was adopted that could minimize both the conditioning and the learning processes, being crucial mechanisms underlying placebo effect. ${ }^{40}$ This was achieved by a structured interview that aimed to clarify the study process and to optimize patients' expectation especially about the rTMS sessions. Moreover, the follow-up visits were assessed by independent rater to decrease the bias resulting from the interaction between the patient, treating clinician, and treatment environment. ${ }^{41}$ The long-term efficacy of rTMS is questionable and needs to be elaborated.

In conclusion, chronic migraine is a road less travelled by an effective, yet, time-locked rTMS sessions; however, BTX-A can fill a major unmet need for those patients as an effective and safe preventive strategy that can be offered for those with disabling primary headaches who failed to respond adequately to conventional treatments and those with unacceptable side effects or in whom standard preventive treatments are contraindicated.

\section{Disclosure}

The authors report no conflicts of interest in this work.

\section{References}

1. Natoli JL, Manack A, Dean B, Butler Q, Turkel CC, Stovner L, Lipton RB. Global prevalence of chronic migraine: a systemic review. Cephalalgia. 2010;30(5):599-609.

2. El-Sherbiny NA, Masoud M, Shalaby NM, Shehata HS. Prevalence of primary headache disorders in Fayoum Governorate, Egypt. $J$ Headache Pain. 2015;16:85.

3. Blumenfeld AM, Varon SF, Wilcox TK, Buse DC, Kawata AK, Manack A, Goadsby PJ, Lipton RB. Disability, HRQoL and resource use among chronic and episodic migraineurs: results from the International Burden of Migraine Study (IBMS). Cephalalgia. 2011;31(3):301-315.

4. Goadsby PJ. Recent advances in understanding migraine mechanisms, molecules and therapeutics. Trends Mol Med. 2007;13(1):39-44.

5. Lauritzen M. Pathophysiology of the migraine aura. The spreading depression theory. Brain. 1994;117(Pt 1):199-210.

6. Zhang X, Levy D, Kainz V, Noseda R, Jakubowski M, Burstein R. Activation of central trigeminovascular neurons by cortical spreading depression. Ann Neurol. 2011;69(5):855-865.

7. Aurora SK, Wilkinson F. The brain is hyperexcitable in migraine. Cephalalgia. 2007;27(12):1442-1453.

8. Vikelis M, Mitsikostas DD. The role of glutamate and its receptors in migraine. CNS Neurol Disord Drug Targets. 2007;6(4):251-257.

9. Brighina F, Palermo A, Fierro B. Cortical inhibition and habituation to evoked potentials: relevance for pathophysiology of migraine. $J$ Headache Pain. 2009;10(2):77-84.

10. Chen W-T, Wang S-J, Fuh J-L, Lin C-P, Ko Y-C, Lin Y-Y. Peri-ictal normalization of visual cortex excitability in migraine: an MEG study. Cephalalgia. 2009;29(11):1202-1211.
11. Zaghi S, Thiele B, Pimentel D, Pimentel T, Fregni F. Assessment and treatment of pain with non-invasive cortical stimulation. Restor Neurol Neurosci. 2011;29(6):439-451.

12. Lipton RB, Pearlman SH. Transcranial magnetic simulation in the treatment of migraine. Neurotherapeutics. 2010;7(2):204-212.

13. Coppola G, Di Lorenzo C, Serrao M, Parisi V, Schoenen J, Pierelli F. Pathophysiological targets for non-pharmacological treatment of migraine. Cephalalgia. Epub 2015 Dec 3.

14. Headache Classification Committee of the International Headache Society (IHS). The International Classification of Headache Disorders, 3rd edition (beta version). Cephalalgia. 2013;33(9):629-808.

15. Beck AT, Ward CH, Mendelson M, Mock J, Erbaugh J. An inventory for measuring depression. Arch Gen Psychiatry. 1961;4:561-571.

16. Jacobson GP, Ramadan NM, Aggarwal SK, Newman CW. The Henry Ford Hospital Headache Disability Inventory (HDI). Neurology. 1994;44(5):837-842.

17. Kosinski M, Bayliss MS, Bjorner JB, et al. A six-item short-form survey for measuring headache impact: The HIT-6TM. Qual Life Res. 2003;12:963-974.

18. Price DD, McGrath PA, Rafii A, Buckingham B. The validation of visual analogue scales as ratio scale measures for chronic and experimental pain. Pain. 1983;17(1):45-56.

19. Blumenfeld A, Silberstein SD, Dodick DW, Aurora SK, Turkel CC, Binder WJ. Method of injection of onabotulinumtoxina for chronic migraine: a safe, well-tolerated, and effective treatment paradigm based on the PREEMPT clinical program. Headache. 2010;50(9): 1406-1418.

20. Padberg F, Zwanzger P, Keck ME, et al. Repetitive transcranial magnetic stimulation (rTMS) in major depression: relation between efficacy and stimulation intensity. Neuropsychopharmacology. 2002;27(4): 638-645.

21. Brighina F, Palermo A, Daniele O, Aloisio A, Fierro B. High-frequency transcranial magnetic stimulation on motor cortex of patients affected by migraine with aura: a way to restore normal cortical excitability? Cephalalgia. 2010;30:46-52.

22. Brighina F, Piazza A, Vitello G, Aloisio A, Palermo A, Daniele O, Fierro B. rTMS of the prefrontal cortex in the treatment of chronic migraine: a pilot study. J Neurol Sci. 2004;227(1):67-71.

23. Misra UK, Kalita J, Bhoi SK. High-rate repetitive transcranial magnetic stimulation in migraine prophylaxis: a randomized, placebo-controlled study. J Neurol. 2013;260(11):2793-2801.

24. Teepker M, Hötzel J, Timmesfeld N, et al. Low-frequency rTMS of the vertex in the prophylactic treatment of migraine. Cephalalgia. 2010;30(2):137-144.

25. Teo WP, Kannan A, Loh PK, Chew E, Sharma VK, Chan YC. Poor tolerance of motor cortex rTMS in chronic migraine. J Clin Diagn Res. 2014;8(9):MM01-MM02.

26. Rossi S, Hallett M, Rossini PM, Pascual-Leone A. Safety, ethical considerations, and application guidelines for the use of transcranial magnetic stimulation in clinical practice and research. Clin Neurophysiol. 2009;120:2008-2039.

27. Welch KM, Barkeley GL, Tepley N, Ramadan NM. Central neurogenic mechanisms of migraine. Neurology. 1993;43(Suppl 3):S21-S25.

28. Afra J, Mascia A, Gérard P, Maertens de Noordhout A, Schoenen J. Interictal cortical excitability in migraine: a study using transcranial magnetic stimulation of motor and visual cortices. Ann Neurol. 1998;44(2): 209-215.

29. Maizels M, Aurora S, Heinricher M. Beyond neurovascular: migraine as a dysfunctional neurolimbic pain network. Headache. 2012; 52(10):1553-1565.

30. Merabet LB, Theoret H, Pascual-Leone A. Transcranial magnetic stimulation as an investigative tool in the study of visual function. Optom Vis Sci. 2003;80(5):356-368.

31. Brigo F, Storti M, Nardone R, Fiaschi A, Bongiovanni LG, Tezzon F, Manganotti P.Transcranial magnetic stimulation of visual cortex in migraine patients: a systematic review with meta-analysis. JHeadache Pain. 2012;13: 339-349. 
32. Conforto A, Amaro E, Gonçalves A, et al. Randomized, proof-ofprinciple clinical trial of active transcranial magnetic stimulation in chronic migraine. Cephalalgia. 2014;34(6):464-472.

33. Leung A, Fallah A, Shukla S, et al. rTMS in alleviating mild TBI related headaches - a case series. Pain Physician. 2016;19:E347-E353.

34. Garcia-Larrea L, Peyron R, Mertens P, et al. Electrical stimulation of motor cortex for pain control: a combined PETscan and electrophysiological study. Pain. 1999;83:259-273.

35. Lorenz J, Cross D, Minoshima S, Morrow T, Paulson P, Casey K. A unique representation of heat allodynia in the human brain. Neuron. 2002; 35:383-393.

36. Lorenz J, Minoshima S, Casey KL. Keeping pain out of mind: the role of the dorsolateral prefrontal cortex in pain modulation. Brain. 2003; 126:1079-1091.
37. Hodaj H, Alibeu JP, Payen JF, Lefaucheur JP. Treatment of chronic facial pain including cluster headache by repetitive transcranial magnetic stimulation of the motor cortex with maintenance sessions: a naturalistic study. Brain Stimul. 2015;8(4):801-807.

38. O'Connell NE, Wand BM, Marston L, Spencer S, DeSouza LH. Noninvasive brain stimulation techniques for chronic pain. Cochrane Database Syst Rev. 2014;4:CD008208.

39. Maizey L, Allen CP, Dervinis M, et al. Comparative incidence rates of mild adverse effects to transcranial magnetic stimulation. Clin Neurophysiol. 2013;124:536-544.

40. Colloca L, Benedetti F. How prior experience shapes placebo analgesia. Pain. 2006;124(1-2):126-133.

41. Finniss DG, KaptchukTJ, Miller F, Benedetti F. Placebo effects: biological, clinical and ethical advances. Lancet. 2010;375(9715):686-695.
Journal of Pain Research

\section{Publish your work in this journal}

The Journal of Pain Research is an international, peer reviewed, open access, online journal that welcomes laboratory and clinical findings in the fields of pain research and the prevention and management of pain. Original research, reviews, symposium reports, hypothesis formation and commentaries are all considered for publication

\section{Dovepress}

The manuscript management system is completely online and includes a very quick and fair peer-review system, which is all easy to use. Visit http://www.dovepress.com/testimonials.php to read real quotes from published authors. 